\title{
Effects of a structured exercise therapy on cancer-related fatigue during and after paediatric stem cell transplantation: a randomized controlled trial
}

\author{
DOI: https://doi.org/10.5114/pq.2021.107847 \\ Michael W. Jung ${ }^{1}$, Susanne Wallek², Anna Senn-Malashonak ${ }^{2}$, Patric Schubert ${ }^{3}$, Klaus Siegler ${ }^{2}$, \\ Andreas Rosenhagen ${ }^{4}$, Winfried Banzer ${ }^{4}$, Peter Bader ${ }^{2}$ \\ ${ }^{1}$ Fresenius University of Applied Sciences Frankfurt, Frankfurt am Main, Germany \\ ${ }^{2}$ Hospital of the Goethe University Frankfurt am Main, Frankfurt am Main, Germany \\ ${ }^{3}$ Fresenius University of Applied Sciences Idstein, Idstein, Germany \\ ${ }^{4}$ Goethe University Frankfurt am Main, Frankfurt am Main, Germany
}

\section{Abstract}

Introduction. Exercise therapy is a frequently applied method in paediatric oncology but there is insufficient evidence for its effectiveness in paediatric stem cell transplantation.

Methods. In a single-centre randomized controlled trial, the effect of exercise therapy (intervention group) was compared with concentration and relaxation techniques (control group). Overall, 72 children were recruited before transplantation and randomly assigned into these 2 groups. They received exercise therapy during and after their hospital stay. A total of 47 inpatient children (age: 6-18 years) were evaluated in phase I: 21 in the intervention and 26 in the control group. The expression of cancer-related fatigue before and after therapy was documented by using the PedsQL 3.0 Multidimensional Fatigue Scale.

Results. All patients deteriorated with regard to fatigue during their hospital stay. A total of 23 outpatient children (stratified into 4 groups) who continued or started exercise therapy showed a significant improvement over 3 and 6 months (phase II) after discharge from hospital (total fatigue score: $p<0.001$, general fatigue: $p=0.002$, sleep and rest fatigue: $p<0.001$ ).

Conclusions. Inpatient children showed no positive effects of exercise therapy on cancer-related fatigue. After discharge, the children in exercise therapy attained better physical constitution. Exercise therapy is effective for successful rehabilitation and outpatient reintegration and therefore recommended to reduce cancer-related fatigue.

Key words: cancer-related fatigue, paediatric oncology, childhood cancer, physical therapy, stem cell transplantation, rehabilitation

\section{Introduction}

Cancer-related fatigue (CRF), which is considered as one of the most bothersome side effects of haematological and oncological diseases, has hardly been researched in German children. Studies from the USA and Great Britain demonstrate the existence of this phenomenon in paediatric oncology [1]. Even if the exact cause of CRF has not yet been clarified, Repka and Hayward [2] pointed out that 'significant correlations between changes in fatigue and both protein oxidation and antioxidant capacity indicate that systemic oxidative stress may be a potential mechanism for CRF'. A changed blood count and a weakened immune system are also factors suspected to cause fatigue. Assuming these hypotheses, exercise therapy could help to reduce CRF. Maybe that was one of the reasons why research on exercise therapy and CRF was started at the beginning of the $21^{\text {st }}$ century.

For example, a study by Hinds et al. [3] involved children aged 7-18 years with a solid tumour or acute myeloid leukaemia. During their hospitalization and chemotherapy, the children were asked to use a bicycle ergometer twice a day for 30 minutes.

Yeh et al. [4] developed a 6-week home training program with aerobics. The intervention group (IG) began the aerobic exercise program 1 week after chemotherapy. A program lasting about 12 weeks was examined by Takken et al. [5]. It included exercises that were home-based and carried out in a community-based physiotherapy practice. Children aged 6-14 years and treated for acute lymphoblastic leukaemia implemented a training program twice weekly at a physiotherapy practice and twice at home.

Randomized controlled trials (RCTs) and meta-analyses in adults examined the influence of exercise therapy and pharmacological interventions on fatigue during and after cancer therapy [6, 7]. Bhardwaj and Koffman [8] pointed out the importance of non-pharmacological interventions for managing fatigue among children. In their literature review, most studies were performed during treatment or during a hospital stay, but none of them showed statistically significant results.

Morales et al. [9] describe in their meta-analysis the effect of exercise training on improving functional mobility of children during and after cancer treatment. An important result is that exercise therapy during treatment does not increase the risk of mortality, recurrence, or adverse effects. In a review by Radossi et al. [10], many forms of traditional and complementary medicine were applied to children, but most of the studies had poor to fair quality. Another study evaluated the effect of a home-based exercise intervention in childhood cancer survivors [11].

More and more RCTs are addressing the question of what effect exercise therapy has on physical fitness and CRF. Lam et al. [12] investigated the effectiveness of an integrated program: the IG demonstrated significantly lower levels of CRF.

Correspondence address: Michael W. Jung, Fresenius University of Applied Sciences Frankfurt, Marienburgstr. 6, D-60528 Frankfurt, Germany, e-mail: jung.michael@web.de 
Braam et al. [13] only found a significant long-term improvement in lower body muscle strength in the IG compared with the control group (CG).

These studies show the importance and possibilities of helping inpatient and outpatient children with exercise therapy, but the data on physiotherapy or exercise therapy in hematopoietic stem cell transplantation (HSCT) are scarce. HSCT is a world-renowned therapy for curative care of different paediatric haematological and oncological diseases. It is also associated with numerous side effects, e.g. nausea, mucositis, neutropenia, and CRF [14-16]. Up to several years after treatment, the recipients experience CRF, which affects their quality of life and participation in daily activities [17-19]. Exercise therapy seems to be a tool to minimize or even prevent these side effects of the disease. Van Haren et al. [20] evaluated 11 RCTs in adult HSCT patients in a meta-analysis. A valuable effect regarding physical functioning, medical outcomes, and psychological variables were found prior to, during, and after therapy [21-24]. In 2011, exercise therapy during paediatric HSCT was evaluated: 13 children were assigned to an IG in which a special exercise program was carried out. This exercise training was shown to be feasible, safe, and promising in the context of health-related quality of life and CRF results [25].

The aim of the present study is to evaluate the impact of exercise therapy on CRF in children and adolescents undergoing HSCT. This leads to the following research questions:

1. Does exercise therapy reduce CRF in paediatric patients after HSCT (the main research question)?

2. Does exercise therapy reduce CRF more than the control condition?

3. When does the intervention most effectively reduce CRF?

\section{Subjects and methods}

This RCT examined both the short- and medium-term effects of a structured exercise therapy during and after HSCT in children and adolescents. The entire study measured the impact of exercise therapy on health-related quality of life, muscle strength, endurance, and CRF.

Data were collected between January 2011 and December 2014 at the Stem Cell Transplantation Centre of the Department of Paediatric and Adolescent Medicine of the Goethe University Hospital Frankfurt, Germany.

It was ethically difficult to justify using an untreated CG. The ethical concerns about an untreated CG result from comparative study results that suggest the success of the intervention. To eliminate the concern that social contacts hinder the results of the experiment, the CG received social contact and human empathy as well. This ensures that the only difference between the 2 groups was the therapy provided to the IG.

This study was a monocentric RCT within 2 phases after HSCT: an inpatient (phase I) and an outpatient (phase II) phase are distinguished. At the beginning of phase I, the participants were randomly assigned to IG or CG. The computerassisted randomization was performed by an internal study manager and was subsequently discussed in detail with patients and their parents.

Phase I observed patients from the acute phase of transplantation inpatient admission (tpre) to discharge (tpost). During inpatient care, both groups received therapy 5 times per week for 45-60 minutes.

The IG received an age-appropriate, individualized treatment that included endurance, strength, and flexibility training.
For the endurance training, the patients with height $\geq 136 \mathrm{~cm}$ used a bicycle ergometer. Given a target exposure time of 10-30 minutes, they self-determined the resistance in training. If a continuous 10-minute loading period was not possible, this should be achieved cumulatively by using the interval method. Children who fell below the minimum body size performed alternative endurance-oriented exercises, such as step aerobics, ball games, moving stories or endurance games with the Nintendo Wii console. To protect against overloading, continuous monitoring of the heart rate was ensured. The objective limit of 180 - age was based on an implementation study [26].

Strength training was aimed at promoting mobility related to activities of daily living and muscle strength. It focused on exercises for the lower extremities and trunk muscles (5 times per week, 3-6 exercises, 1-3 sets, 8-15 repetitions, breaks of $60-120$ seconds). The individual load intensity was dosed with small devices and exercise modifications. Tasks were taught outside the bed, but with reduced health or treatment limitations, a bed-adapted program was used.

The flexibility training included active and passive stretching. To avoid injuries as well as haemorrhages, mainly static exercises with slight intensities were recommended.

The CG received age-specific concentration training (memorial/mental training, e.g. jigsaw), perceptual and relaxation exercises. The training was implemented every day for 45-60 minutes as well, so that these children also had the social benefit of the treatment. Each day, a low-mobility intervention (e.g. attention and concentration training, relaxation elements) was performed.

In view of the increasing prevalence of long-term consequences, especially after therapy specific to the central nervous system, the 45-60-minute control intervention included predominantly a cognitive promotion:

- This involved riddles, knowledge quizzes, parlour games with cognitive pretension, perceptual exercises, etc.

- The second part of the training session comprised passive relaxation methods, such as hedgehog ball massages, autogenic training, or a free employment whose content was directed to the individual wishes and inclinations of the patients.

To ensure that the CG did not experience severe mobility limitations, the control intervention was performed outside the bed, at a table, as much as possible. The physiotherapist was able to detect early contractures or muscular atrophies and initiate necessary measures with the ward physicians.

In phase II of the study, the participants were examined at day +100 (t100: 3-month follow-up) and at day +200 (t200: 6-month follow-up). Owing to the special requirements for ambulatory follow-up care, a new stratification of the subjects was necessary (shown in Figure 1). The participants who were previously randomized by a computer into 1 of the 2 study conditions were now allocated to 4 new groups. Children and adolescents who continued medical care in Frankfurt could participate in an outpatient exercise therapy; all foreign minors were allocated to a wait-control group. The outpatient treatment ended 6 months after HSCT.

The whole study protocol can be accessed at the Department of Stem Cell Transplantation and Immunology, Children's Hospital of the Goethe University Frankfurt.

After written consent was obtained, the children completed a battery of subjective and objective testing to assess physiological and psychometric criteria at least 7 days prior to hospitalization. Sealed, opaque, and sequentially numbered envelopes were opened by the study coordinators after the testing. 


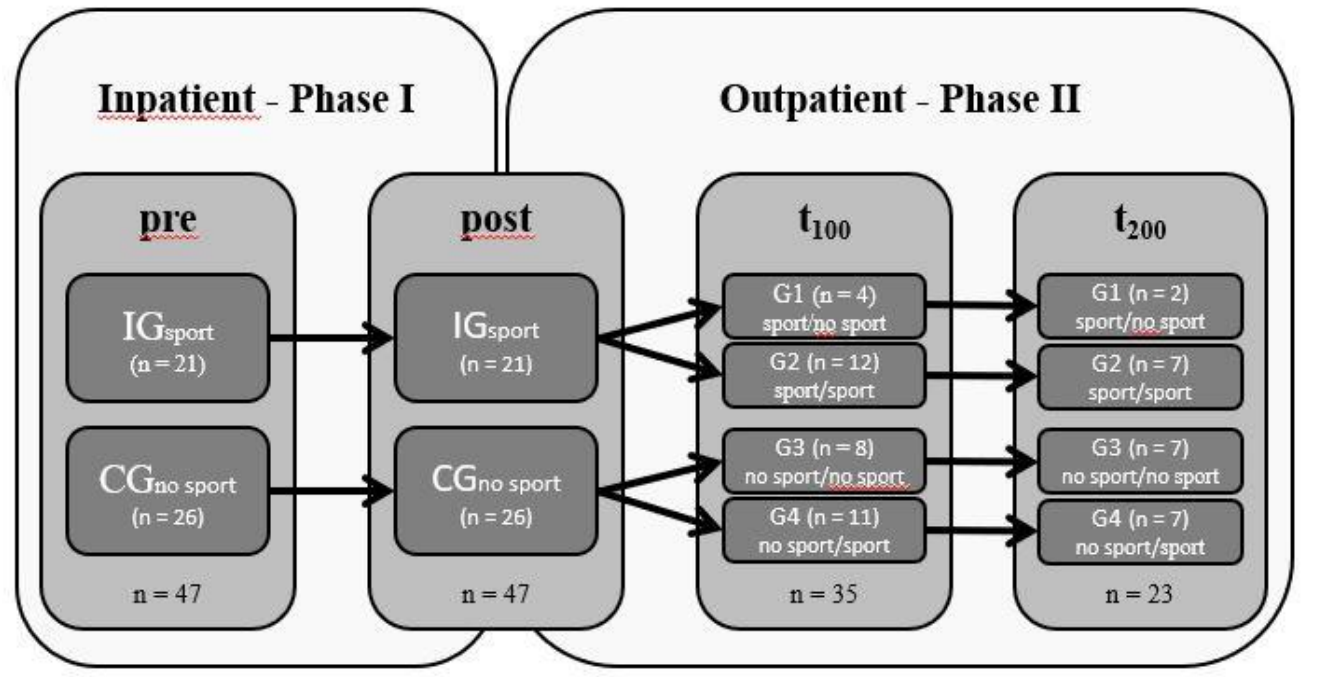

IG - intervention group CG - control group t100 - 3-month follow-up t200 - 6-month follow-up G - group

Figure 1. Participants after randomization (inpatient) and stratification (outpatient)
To investigate the effect of exercise therapy on CRF, the PedsQL 3.0 Multidimensional Fatigue Scale was used [27]. The questionnaire has 3 dimensions, each with 6 questions that add up to the total fatigue score (TFS). A percentile rank of 100 indicates no CRF symptoms; decreasing values indicate increased fatigue. This questionnaire confirms CRF with low values in the dimensions of general fatigue (GF), sleep and rest fatigue (SRF), and cognitive fatigue (CF), so these dimensions were used as primary outcomes. All PedsQL questionnaires were evaluated by a blinded reviewer at the end of the study.

The study involved a total of 72 children and adolescents (24 girls, 48 boys) aged 5-18 years (mean: 11.1 years) who underwent allogeneic or autologous stem cell transplantation.

No power calculation was performed. Recruitment was based on the inclusion and exclusion criteria as part of the medical preparation for transplantation by the physicians.

The inclusion criteria were as follows: written informed consent provided by the parents, age of at least 5 years, an indication for allogeneic or autologous stem cell transplantation, basic knowledge of German or English (both children and their parents).

The exclusion criteria involved age $<5$ or $>18$ years, contraindications for exercise, language barriers, indications for specific physiotherapeutic treatment during transplantation, as well as severe orthopaedic, neurological, or cardiopulmonary comorbidities.

The study participants and their parents were informed in detail verbally and in writing about the objectives of the study by a physician. Participation withdrawal was possible at any time without giving reasons and regardless of the medical therapy effects.

After excluding subjects from analysis (e.g. missing values), the IG comprised 21 and the CG 26 subjects. The sample consisted of 18 girls and 29 boys with different tumours as shown in Table 1.

In phase II, the 2 groups were stratified in accordance with the participants' proximity to our centre. Children who lived far away received their follow-up examinations in their home clinics (which could not perform HSCT). Children living close to our centre were offered an outpatient sports program with the same content as the IG. Phase II began right after hospital discharge (tpost) and started with a stratified sample of 4 groups. These participants received on average about $1.5 \times$ weekly training sessions of 30-50 minutes (mean: 40 minutes). The more distant-living children received only no exercise therapy. Whether or not they started sports activities privately, they did not report during the consultation.

This led to the following new grouping:

- group 1: inpatient sports and outpatient no sports $(n=4)$, - group 2: inpatient sports and outpatient sports $(n=12)$,

- group 3: inpatient no sports and outpatient no sports $(n=8)$,

- group 4: inpatient no sports and outpatient sports $(n=11)$.

Group homogeneity was tested in phase I via an independent-measures $t$-test of the pre-total scores, and in phase II via one-way ANOVA of post-total scores considering both samples (owing to exclusions within the repeated measurement design). Differences were analysed by using typical repeated-measures statistical methods and respective group variables were included as between-subject factors. Therefore, the hypotheses were arranged with a 2-factorial ANOVA approach (2 main effects and 1 interaction effect). Considering tpre and tpost, a $2 \times 2$ ANOVA was performed with the dichotomous group variable (IG, CG). Further analyses to find differences within the testing times tpost, t100, and t200 were conducted by using a $3 \times 4$ ANOVA owing to the new stratification (groups 1-4).

We differentiated between sub-hypotheses regarding each subscore of the questionnaire.

The Mauchly's and correlation tests were performed to verify homoscedasticity. Variance homogeneity was tested via Levene's tests. The significance level was preset to $\alpha=0.05$ (tendency significance defined as $\alpha=0.1$ ).

In addition to these analyses, we carried out an intentionto-treat analysis. Because of our high percentage of missing data, a mixed model approach without any ad-hoc imputation is more powerful than other options.

The CONSORT flow diagram of the study is presented in Figure 2.

\section{Ethical approval}

The research related to human use has complied with all the relevant national regulations and institutional policies, has followed the tenets of the Declaration of Helsinki, and has been approved by the ethics committee of the Department of Medicine at the Goethe University Frankfurt. The study is registered at http://www.clinicaltrials.gov under the identification number NCT01575704.

\section{Informed consent}

Informed consent has been obtained from the legal guardians of all individuals included in this study. 
Table 1. Biometrical data of the samples in different experimental phases

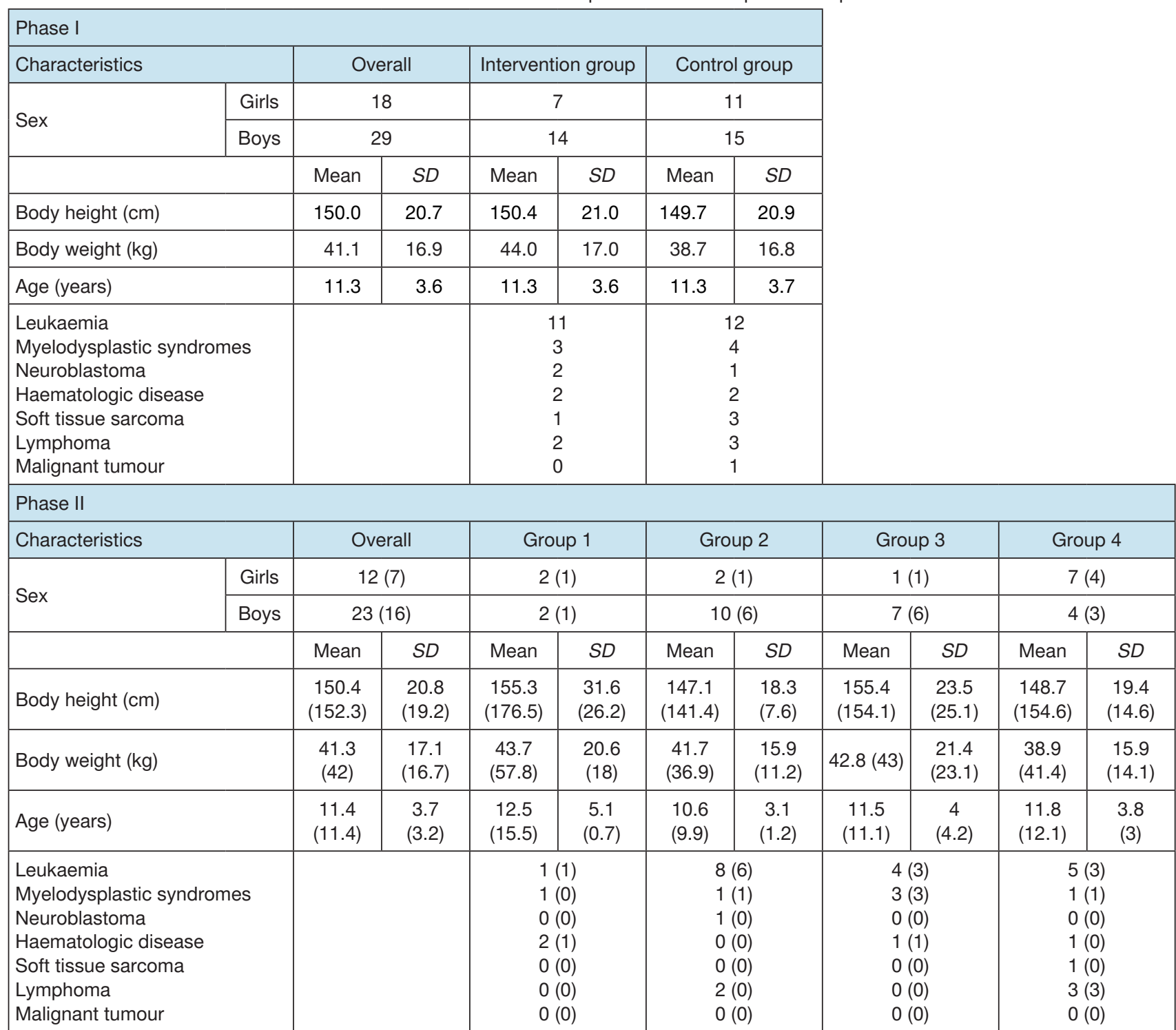

Numbers in brackets represent the smaller sample size in t200 (6-month follow-up)

\section{Results}

Only data on CRF are presented in this paper. Despite the initial scepticism of the patients, parents, and staff, there was a particularly good acceptance of the intervention.

With regard to group homogeneity, no differences in the total score were found either in phase I (tpre score: T_45 = $1.618, p=0.113$ ) or in phase II (tpost score, group t100: $\bar{F}_{3,34}=$ $0.706, p=0.555$; tpost score, group t200: $F_{3,22}=0.12, p=$ 0.947). Detailed patient characteristics are shown in Table 1.

Throughout the study, there were some dropouts due to complications, patient death (31.6\% each), and missing values. In phase I, the dropout rate was $50 \%$, so we started phase II with 35 children and finished the study with the data of 23 children (32.9\%).

The reasons for the study dropouts were the death of the subjects, changes in health status, and difficulties with the questionnaire. Therefore, only 23 patients could be considered for the analysis of the effect of exercise therapy in outpatient children.

The IG had an average of 3.1 ( \pm 0.6 , range: $2-4$ ) training sessions per week in phase $\mathrm{I}$, with a mean training duration of $50.3( \pm 6.3)$ minutes per training session. The ratings of perceived exertion averaged $13.9( \pm 1.2)$, which indicates that the training was somewhat exhausting. The CG received 2.9 units per week (range: $0.3-4$ ) with 51 minutes per training unit (range: $30-60$ minutes). The visible and measurable impact on CRF was determined as follows.

In TFS, GF, and SRF, a general trend was that median values decreased in the intervention phase (from tpre to tpost): TFS: -16.67 median points (Mp), GF: $-14.58 \mathrm{Mp}$, SRF: $-37.5 \mathrm{Mp}$. The reduced values indicate increased fatigue. Clearly recognizable in both groups is a marked decrease in TFS from tpre to tpost. As shown in Figure 3, in the IG, a significant decrease of CRF values from tpre to tpost is obvious.

For the inpatient phase I, the main research question cannot be confirmed.

In the follow-up measurements (t100, t200), an increase to the base level was obvious (shown in Figures 4 and 5). Both groups exhibited a tendency toward a significant reduction in CRF; the IG demonstrated a significant reduction 6 months after clinical discharge.

Regarding $2 \times 2$ ANOVA in the case of the main effect tpre vs. tpost and 2 groups, we found significant overall results in the measures of TFS $\left(F_{1,45}=16.79, p<0.001, \eta^{2}=0.27\right)$, $\operatorname{SRF}\left(F_{1,45}=27.44, p<0.001, \eta^{2}=0.38\right)$, and GF $\left(F_{1,45}=11.59\right.$, $\left.p=0.001, \eta^{2}=0.2\right)$. No significant effect was observed in CF $\left(F_{1,45}=1.87, p=0.18\right)$. 


\section{Inpatient (phase I: pre-post)}

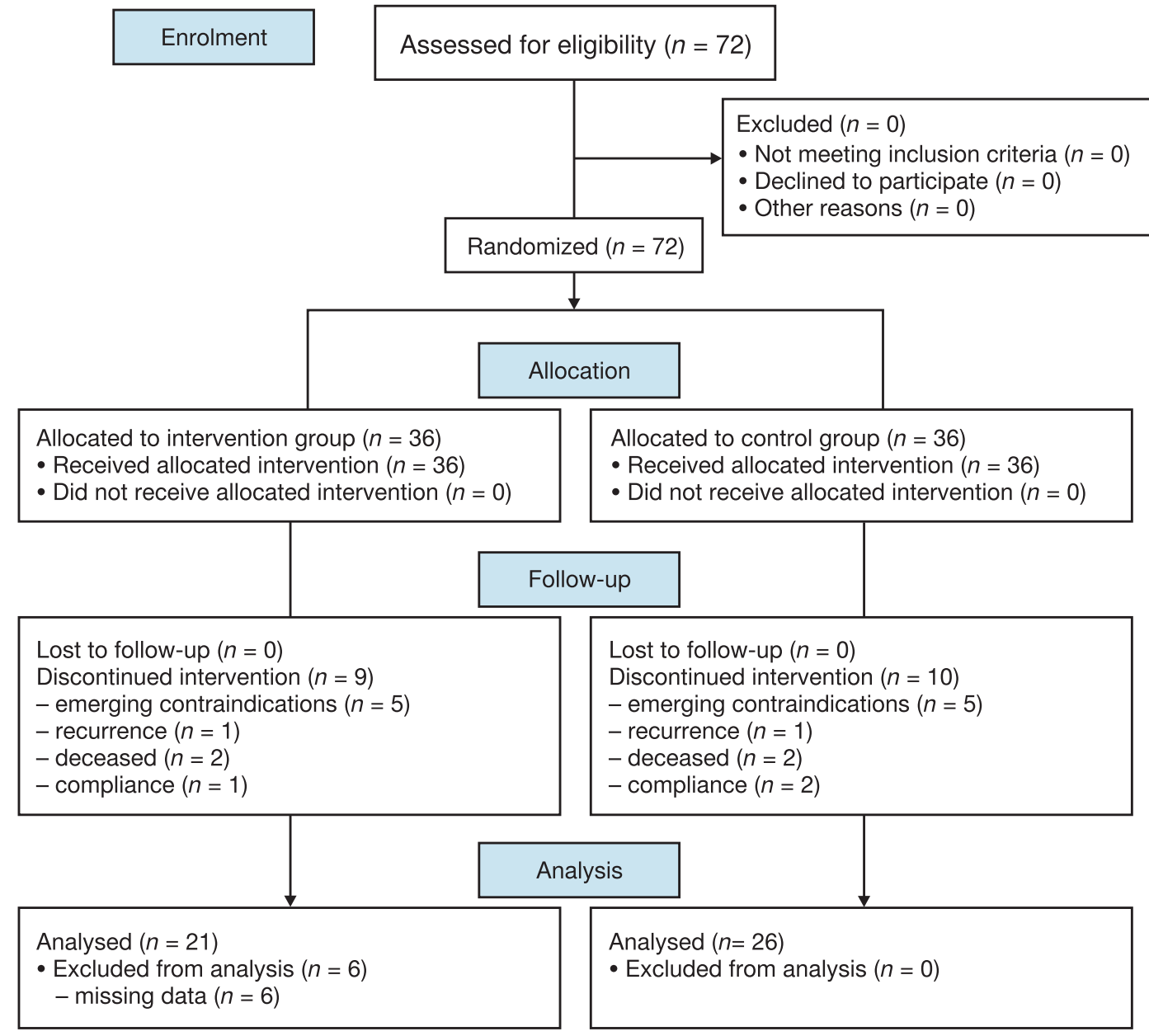

\section{Outpatient (phase II: post-t200)}

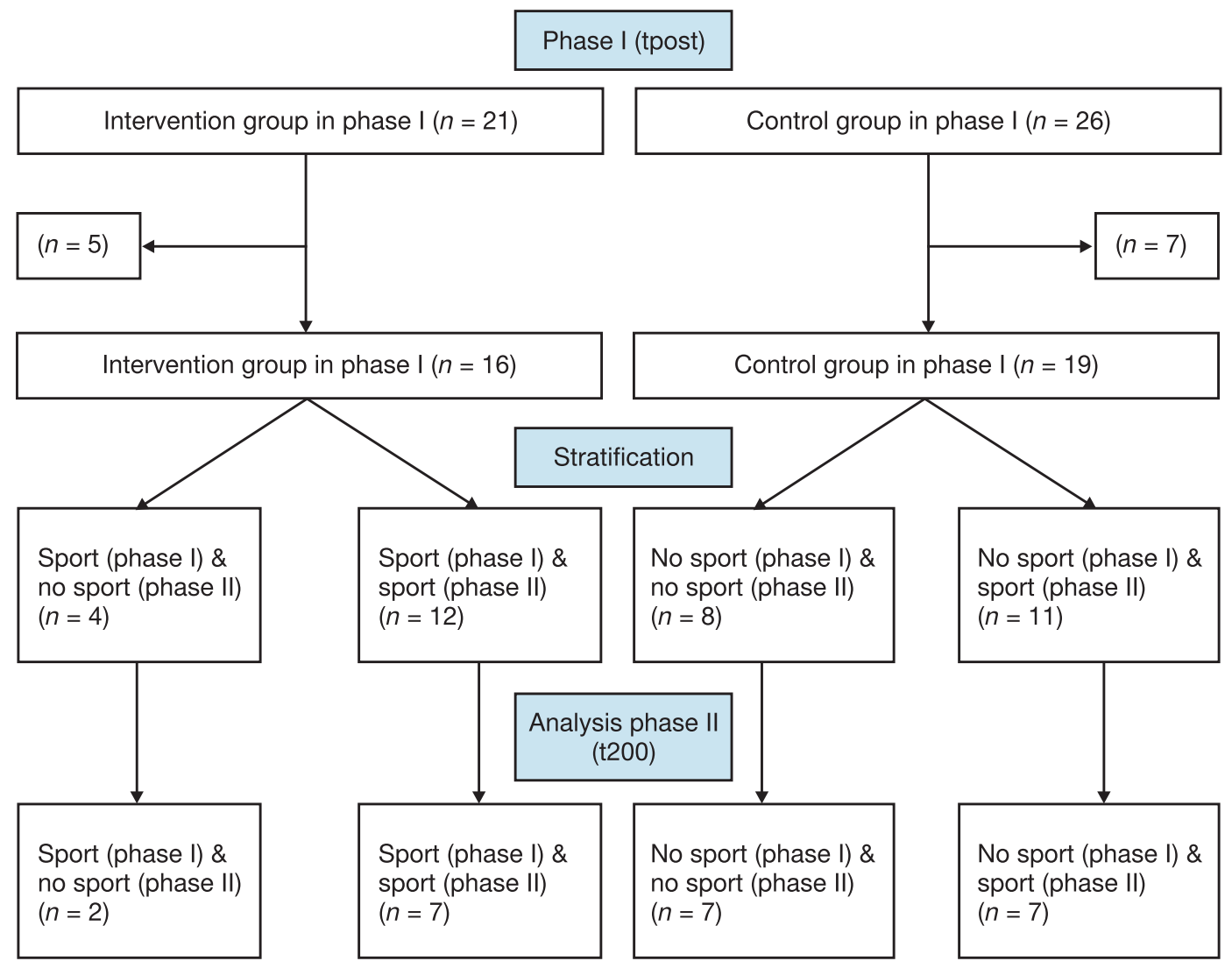

Figure 2. CONSORT flow diagram of the study 

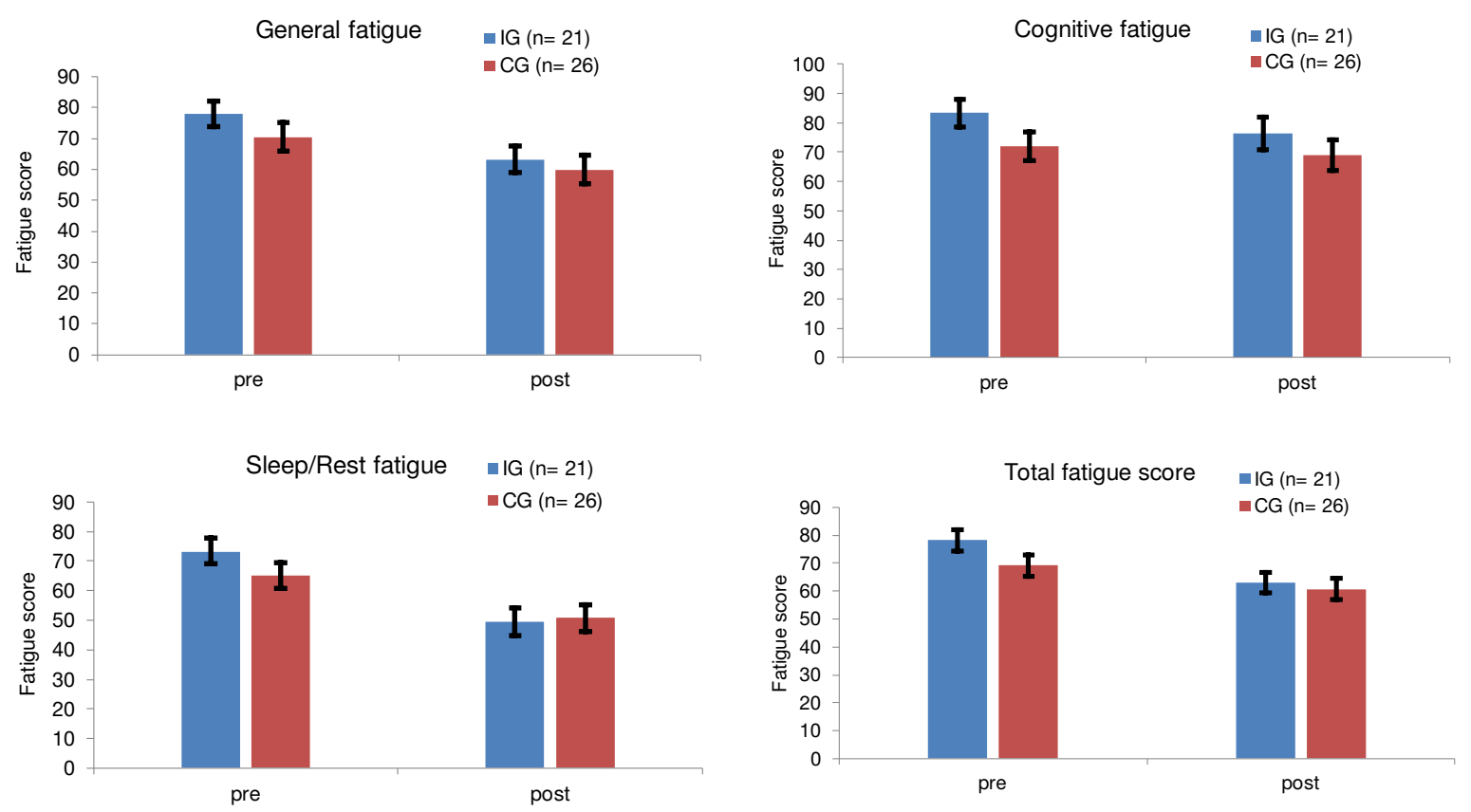

IG - intervention group, CG - control group

Figure 3. Phase I: plots by subscores and groups (low values mean high fatigue)

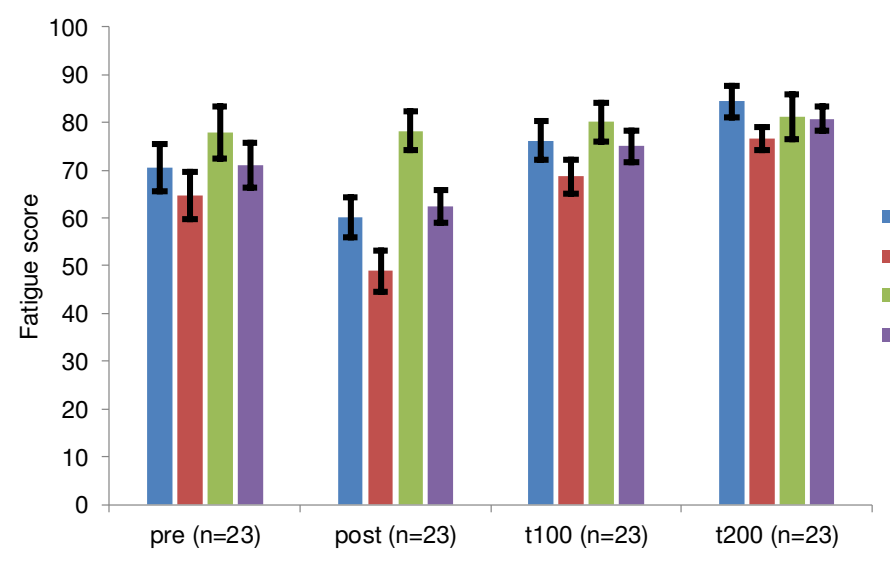

- General fatigue

- Sleep/Rest fatigue

- Cognitive fatigue

- Total fatigue score
Figure 4. Phase II: main effect over time
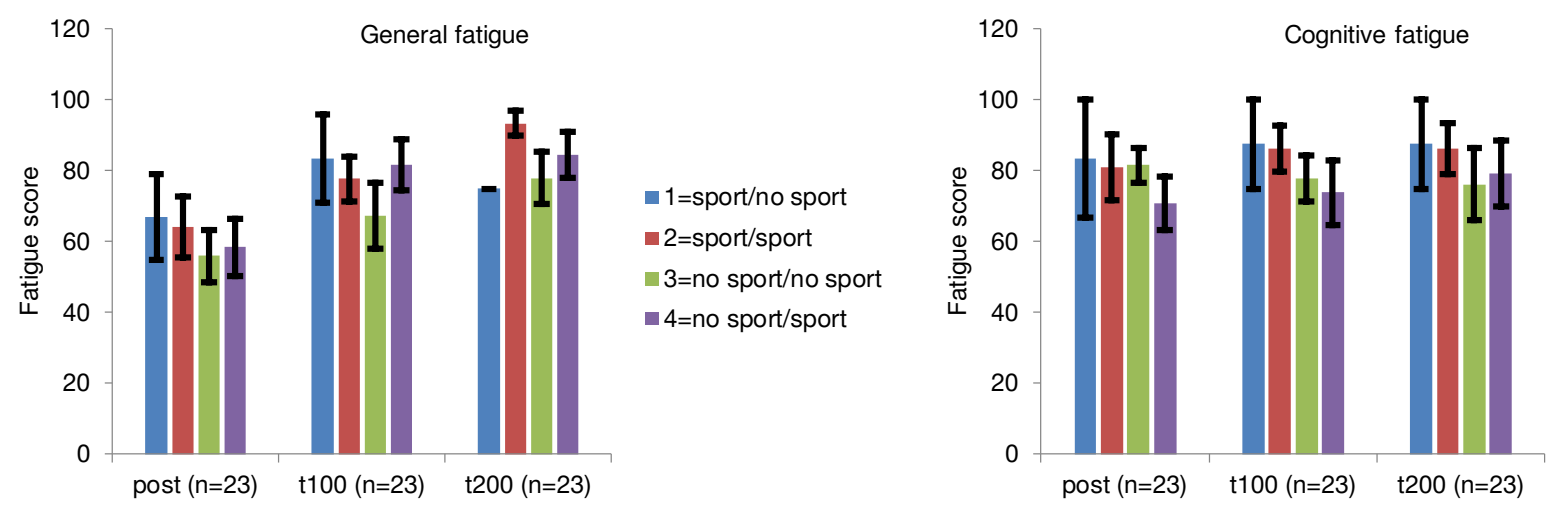

-1=sport/no sport

-2=sport/sport

- $3=$ no sport/no sport

घ4=no sport/sport

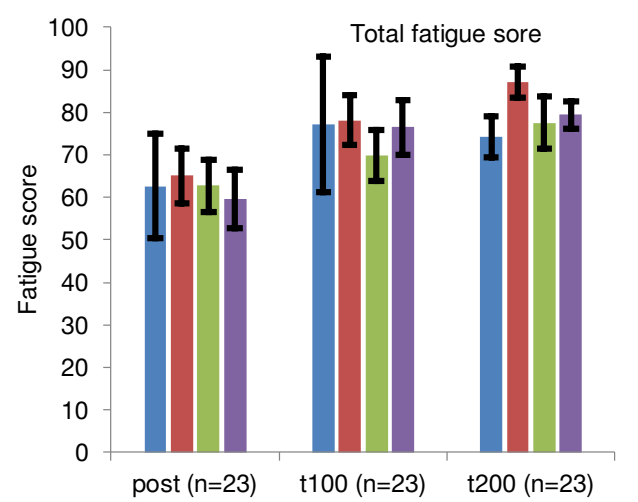

-1=sport/no sport

— $2=$ sport/sport

- $3=$ no sport/no sport

4= no sport/sport

$3=$ no sport/no sport

-4=no sport/sport

post $(n=23) \quad t 100(n=23) \quad t 200(n=23)$

Figure 5. Phase II: plots by subscores and groups 
The randomized IG displayed a significant improvement in their fatigue values at $\mathrm{t} 100$ and a significant improvement in their fatigue values at t200 compared with the inpatient values. These results fit the meta-analysis by Oberoi et al. [28].

Children randomized to the CG also improved at $\mathrm{t} 100$ and $t 200$, but less distinctly than those in the IG.

An analysis of the GF, SRF, and CF dimensions revealed the following:

- The GF dimension was remarkably like TFS: an outpatient improvement at $\mathrm{t} 100$ and t200 was more visible in the IG than in the $C G$.

- The SRF dimension demonstrated significant improvements in the IG at t100 and significant improvements at t200 after the significant inpatient deterioration. The CG improved but not significantly.

- In the CF dimension, no improvement was reported.

Furthermore, the IG was not significantly different from the $C G$ in each variable; however, the IG presented a tendency of greater values in fatigue scales consistently over all subscores (TFS: $13 \%$, GF: $10 \%$, SRF: $12.5 \%$, CF: $15.7 \%$ ). The interactions were shown to be not significant (TFS: $p=$ 0.25 , GF: $p=0.58$, SRF: $p=0.2$, CF: $p=0.59$ ).

Within phase II, ANOVA revealed a significant main effect over time in TFS $\left(F_{2,38}=9.73, p<0.001, \eta^{2}=0.34\right)$, GF $\left(F_{2,38}=\right.$ 9.14, $\left.p=0.002, \eta^{2}=0.33\right), \operatorname{SRF}\left(F_{2,38}=12.63, p<0.001, \eta^{2}=\right.$ $0.4)$, but not in $\operatorname{CF}\left(F_{2,38}=0.3, p=0.74\right)$, as shown in Figure 4 .

Differences between groups turned out not significant. Because of group homogeneity after stratification, in tpost, the ranges in absolute score points were 5.4 in TFS, 11.1 in GF, 13.1 in SRF, and 12.5 in CF (Figure 4). The interaction evaluation showed no significant effect in each variable (TFS: $p=0.89$, GF: $p=0.7$, SRF: $p=0.93$, CF: $p=0.83$ ). The second research question cannot be confirmed for inpatient children.

The group means over time are presented in Figure 5. After the stratification (phase II), the 4 groups were evaluated on the basis of their sporting behaviour.
Owing to the recognizable results in TFS, the following statements can be made:

- Children who were engaged in sports in phase I but stopped exercising after hospital discharge (group 1) experienced little recovery from fatigue at the end of the study.

- Children who consistently engaged in sports during the study period (group 2) showed the greatest reduction in fatigue at $\mathrm{t} 100$ and $\mathrm{t} 200$.

- Children who did not engage in sports during the entire study period (group 3 ) exhibited only a minimal recovery from fatigue.

- Children who were inpatient in the CG and became active in phase II (group 4) presented a marked reduction in fatigue at $\mathrm{t} 100$ and $\mathrm{t} 200$.

The third research question shows that significant CRF reduction occurs only after discharge when patients are exercising. This remains only partly in line with a study by Witlox et al. [29], who described positive short- and long-term effects on CRF.

A closer examination of the dimensions revealed the following. In the GF dimension, a similar picture appeared as in the case of TFS. Group 2 had the best outcome at $\mathrm{t} 200$, followed by group 4 . The difference between the 2 weaker groups (1 and 3) was not very distinct here.

Regarding SRF, a somewhat different picture emerges. While group 2 and group 4 continued to present good outcome after clinical discharge, the result of group 1 is noteworthy. Outpatient children who stopped engaging in sports after clinical discharge exhibited the worst values. The values were even worse than those in the children who engaged in no sports as inpatients or outpatients (group 3). In the CF dimension, all children achieved remarkably similar results at each point. During the study period, hardly any changes were observed.

Within an intention-to-treat approach of phase II (post, t100, t200), a linear mixed effects model with time*group (both as fixed factors) and subjects as random factor was

\section{General fatigue}

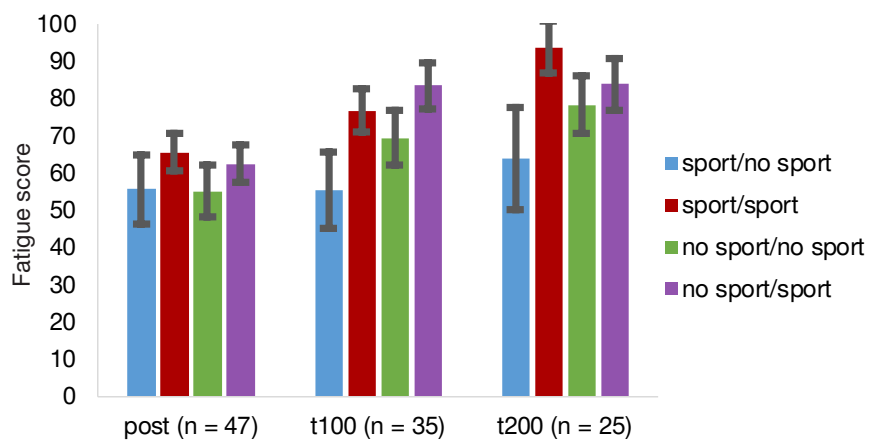

Sleep/Rest fatigue
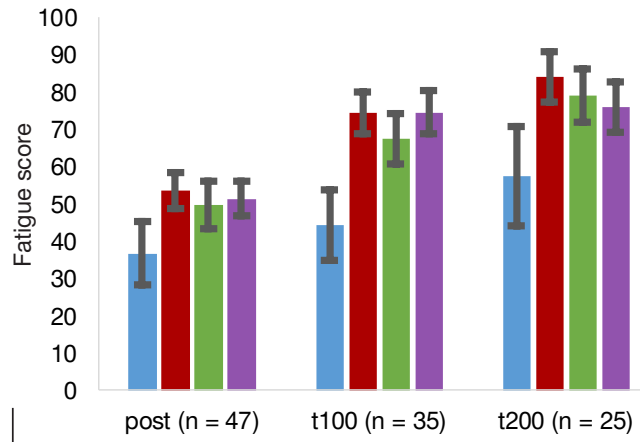

- sport/no sport

- sport/sport

- no sport/no sport

no sport/sport

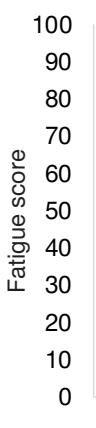

100

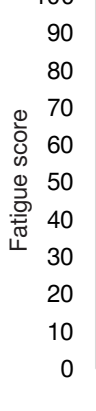

Cognitive fatigue

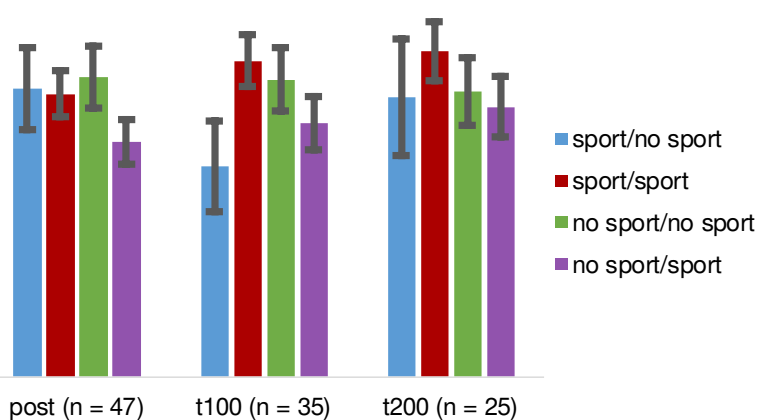

Total fatigue score

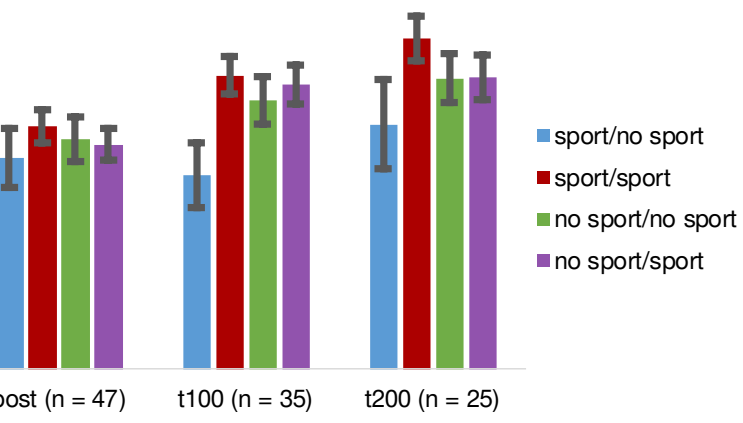

Figure 6. Results of the linear mixed effects model: estimated marginal means of the 4 groups in phase II and standard errors 
Table 2. Results of significance tests with respect to phase I and phase II

\begin{tabular}{|c|c|c|c|c|c|c|}
\hline Phase I $(n=47)$ & \multicolumn{2}{|c|}{ tpre-tpost } & \multicolumn{2}{|c|}{ Groups } & \multicolumn{2}{|c|}{ Interaction } \\
\hline $2 \times 2$ ANOVAa $^{a}$ & $F_{1,45}$ & $p$ & $F_{1,45}$ & $p$ & $F_{1,45}$ & $p$ \\
\hline GF & 11.59 & $<0.01^{*}$ & 1.11 & 0.30 & 0.32 & 0.57 \\
\hline SRF & 27.44 & $<0.01^{*}$ & 0.44 & 0.51 & 1.64 & 0.21 \\
\hline CF & 1.87 & 0.18 & 2.19 & 0.15 & 0.30 & 0.59 \\
\hline TFS & 16.79 & $<0.01^{*}$ & 1.44 & 0.24 & 1.33 & 0.25 \\
\hline Phase II $(n=23)$ & \multicolumn{2}{|c|}{ tpost-t100-t200 } & \multicolumn{2}{|c|}{ Groups } & \multicolumn{2}{|c|}{ Interaction } \\
\hline $3 \times 4$ ANOVA $^{a}$ & $F_{2,38}$ & $p$ & $F_{3,19}$ & $p$ & $F_{6,38}$ & $p$ \\
\hline GF & $9.14^{b}$ & $<0.01^{*, \mathrm{~b}}$ & 0.76 & 0.53 & $0.60^{b}$ & $0.69^{b}$ \\
\hline SRF & 12.63 & $<0.01^{*}$ & 0.89 & 0.47 & 0.31 & 0.93 \\
\hline CF & 0.30 & 0.74 & 0.42 & 0.74 & 0.47 & 0.83 \\
\hline TFS & 9.73 & $<0.01^{*}$ & 0.40 & 0.75 & 0.37 & 0.89 \\
\hline Phase II (ITT analysis) & \multicolumn{2}{|c|}{ tpost-t100-t200 } & \multicolumn{2}{|c|}{ Groups } & \multicolumn{2}{|c|}{ Interaction } \\
\hline 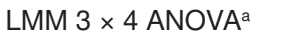 & $\mathrm{Chi}^{2}{ }_{2}$ & $p$ & $\mathrm{Chi}_{3}{ }_{3}$ & $p$ & $\mathrm{Chi}_{6}{ }_{6}$ & $p$ \\
\hline GF & 33.05 & $<0.01^{*}$ & 5.55 & 0.14 & 4.76 & 0.58 \\
\hline SRF & 48.65 & $<0.01^{*}$ & 9.91 & $0.02^{*}$ & 2.13 & 0.91 \\
\hline CF & 1.41 & 0.49 & 4.49 & 0.21 & 6.38 & 0.38 \\
\hline TFS & 29.69 & $<0.01^{*}$ & 5.44 & 0.14 & 4.47 & 0.61 \\
\hline
\end{tabular}

GF - general fatigue

SRF - sleep and rest fatigue

CF - cognitive fatigue

TSF - total fatigue score

ITT - intention-to-treat

LMM - linear mixed model

* significant $p$ values, a variance homogeneity in all groups confirmed, ${ }^{b}$ Greenhouse-Geisser correction

conducted as this procedure may be appropriate to account for the high dropout rate. All fixed effects were analysed with type II Wald $\chi^{\wedge} 2$-tests. Considering the total score, we found a significant effect in time $\left(\chi \_2^{\wedge} 2=29.69, p<0.001\right)$ but not in the time* ${ }^{*}$ roup interaction $\left(\chi 6^{\wedge} 2=4.47, p=0.61\right)$. A similar result was obtained within GF (time: $\chi 2^{\wedge} 2=33.05, p<0.001$; time*group: $\chi 6^{\wedge} 2=4.76, p=0.58$ ) and SRF (time: $\chi \_2^{\wedge} 2=$ 48.65, $p<0.001$; time*group: $\left.\chi \_6^{\wedge} 2=2.13, p=0.91\right)$. With respect to CF, we revealed no effects (time: $\chi \_2^{\wedge} 2=1.41$, $p=0.49$; time*group: $\chi 6^{\wedge} 2=6.38, p=0.38$ ). The estimated marginal means and standard errors are shown in Figure 6.

Table 2 clearly summarizes the results of all essential significance tests.

\section{Discussion}

Regarding other studies, it must be explained that the exercises of this study lasted longer than 30 minutes (median: 51 minutes; range: 30-60 minutes) [1, 3]. Although other studies did not show significant results [8] or ambivalent outcomes [12], the significant results of this study should be discussed against the background of HSCT. It should be borne in mind that this sample is a vulnerable population: inpatient children after HSCT in a state of aplasia, in a progressive deterioration as a transplantation-related degeneration.

To refer to the 'why does it work,' our results coincide with those obtained by Repka and Hayward [2], who showed that exercise therapy led to a more robust and significant in- crease in antioxidant capacity and decrease in protein oxidation, mitigating CRF.

A causal answer is not possible now, but Chamorro-Viña et al. [30] also confirm our hypothesis with 6 children. They point out that after HSCT, natural killer cell cytotoxicity (NKCC) recovery is important for good outcomes. The ratio of NKCC was 8 times greater in their IG. They stated that a moderateintensity exercise therapy was feasible and might redistribute the natural killer cell subset, improving NKCC. In contrast to Repka and Hayward [2], however, they implemented exercise therapy early after HSCT.

Even if a sports program involves certain risks after HSCT, no incidents were identified.

These findings correspond with a meta-analysis performed by Meneses-Echávez et al. [6], who described exercise therapy as safe and effective for improving CRF.

The clinical daily routines are stressful factors which may have influenced the outcome of this study. Another frequently underestimated factor is the deconditioning of physical constitution occurring within HSCT pre-treatment. The conditioning regime is an important determinant of fatigue after transplantation. Mustian et al. [7] indicated in a meta-analysis that the effect of exercise therapy depended on the stage of cancer and the baseline treatment. During and after cancer therapy, exercise and psychological interventions were significantly more effective than pharmaceutical options.

Cumulative toxicities increase the extent to which transplantation is negatively affected and raise the morbidity and mortality risk. Muscular atrophy, neuromuscular deficits, and 
cardiopulmonary impairment are observed in children during and after primary treatment.

Overall, it must be admitted that after stratification into the subgroups, the study involved a small sample. Few HSCT patients completed the study (owing to varied disorders or problems, e.g. high morbidity and mortality risk, non-responders).

With reference to $C F$, the following questions could arise: Does CRF not affect this dimension in children, or do they not correctly reflect their feelings? Maybe the PedsQL 3.0 Multidimensional Fatigue Scale does not adequately assess CF? Are adolescents ashamed of showing weaknesses in this area? The results concerning CF confirm observations from a previous study [27].

Owing to an overlapping training effect from the inpatient exercise therapy in group 1 , they presented moderate values at $\mathrm{t} 100$ but could not improve at $\mathrm{t} 200$ without engaging in sports. The minimal recovery from CRF in group 3 could represent a spontaneous remission without training.

The intention-to-treat analysis largely confirmed the results of the as-treated analysis. In relation to TFS, which exhibited the most significant improvement, the intention-to-treat analysis and the as-treated analysis are $87.5 \%$ identical. Only in the as-treated analysis did groups 1 and 3 swap the last places. For SRF and GF, the intention-to-treat and as-treated analyses for $\mathrm{t} 200$ gave identical results. The improvement of GF in group 1 at $\mathrm{t} 100$ in the as-treated analysis, possibly caused by statistical outliers, was levelled in the intentionto-treat analysis.

Our results, as well as those obtained by Repka and Hayward [2], encourage the current guidelines for cancer survivors, which suggest moderate physical activity as an option to 'reduce treatment-associated side effects, an effect that may be in part due to changes in antioxidant capacity and oxidative stress'.

\section{Limitations}

Despite the promising results, a few study limitations must be mentioned. The HSCT underlying diseases led to a very heterogeneous study population, with different chemotherapy regimens. The difference between allogeneic and autologous transplantation is one aspect that was ignored owing to a small number of cases. Because of a small sample size, it can be assumed that the type II error was high. But this also means that the descriptive tendencies are not negligible. Regarding the significant effects identified, the small sample size is not critical.

In phase II, the sample had a minor gender bias (16 boys, 7 girls) and an age bias between groups 1 and 2 (mean age: 15.5 and 9.9 years, respectively). A follow-up study with a larger number of children is recommended.

\section{Conclusions}

Owing to the low effect during the stationary phase, other applications for reducing side effects can be given priority here. The impressive reduction of CRF after clinical discharge shows how important exercise therapy can be for children in paediatric oncology. Not only the participation in sports and thus a higher quality of life but also the possibly of better functioning at school are feasible with less fatigue. For these reasons, the authors recommend early sporting activities after HSCT, which should be maintained even after clinical discharge in the rehabilitation phase. Because of the small number of cases, the result should only constitute a careful recommendation.

\section{Disclosure statement}

The entire BISON (Bewegungstherapie in der Pädiatrischen Stammzelltransplantation) study received funding by José Carreras Leukaemia Foundation Germany (project No. 10/29), Help for Children with Cancer Association, Hand-inHand for Children with Cancer Altenstadt, Association for Children with Cancer and Chronically III Children Darmstadt, Adolf Messer Foundation. The donations were used to finance 2 positions for sports scientists, several bicycle ergometers, and various small devices.

The corresponding author of this manuscript was an external advisor to the study. He does not have any financial interest and did not receive any financial benefit from this research.

\section{Conflict of interest}

The authors state no conflict of interest.

\section{References}

1. Jung M, Zepf N, Fuchs B. Exercise therapy for children with cancer related fatigue [in German]. Klin Padiatr. 2016;228(3):157-163; doi: 10.1055/s-0042-105291.

2. Repka CP, Hayward R. Effects of an exercise intervention on cancer-related fatigue and its relationship to markers of oxidative stress. Integr Cancer Ther. 2018;17(2): 503-510; doi: 10.1177/1534735418766402.

3. Hinds PS, Hockenberry M, Rai SN, Zhang L, Razzouk BI, Cremer $L$, et al. Clinical field testing of an enhanced-activity intervention in hospitalized children with cancer. J Pain Symptom Manage. 2007;33(6):686-697; doi: 10.1016/j.jpainsymman.2006.09.025.

4. Yeh $\mathrm{CH}$, Man Wai JP, Lin U-S, Chiang Y-C. A pilot study to examine the feasibility and effects of a home-based aerobic program on reducing fatigue in children with acute lymphoblastic leukemia. Cancer Nurs. 2011;34(1):3-12; doi: 10.1097/NCC.0b013e3181e4553c.

5. Takken T, van der Torre $P$, Zwerink M, Hulzebos EH, Bierings M, Helders PJM, et al. Development, feasibility and efficacy of a community-based exercise training program in pediatric cancer survivors. Psychooncology. 2009;18(4):440-448; doi: 10.1002/pon.1484.

6. Meneses-Echávez JF, González-Jiménez E, RamírezVélez R. Effects of supervised exercise on cancer-related fatigue in breast cancer survivors: a systematic review and meta-analysis. BMC Cancer. 2015;15:77; doi: 10.1186/s12885-015-1069-4.

7. Mustian KM, Alfano CM, Heckler C, Kleckner AS, Kleckner IR, Leach CR, et al. Comparison of pharmaceutical, psychological, and exercise treatments for cancer-related fatigue: a meta-analysis. JAMA Oncol. 2017;3(7):961968; doi: 10.1001/jamaoncol.2016.6914.

8. Bhardwaj T, Koffman J. Non-pharmacological interventions for management of fatigue among children with cancer: systematic review of existing practices and their effectiveness. BMJ SupportPalliat Care. 2017;7(4):404414; doi: 10.1136/bmjspcare-2016-001132.

9. Morales JS, Valenzuela PL, Rincón-Castanedo C, Takken T, Fiuza-Luces C, Santos-Lozano A, et al. Exercise training in childhood cancer: a systematic review and meta-analysis of randomized controlled trials. Cancer Treat Rev. 2018;70:154-167; doi: 10.1016/j.ctrv.2018. 08.012 .

10. Radossi AL, Taromina K, Marjerrison S, Diorio CJ, Similio R, Njuguna F, et al. A systematic review of integrative clinical trials for supportive care in pediatric oncology: a report from the International Society of Pediatric 
Oncology, T\&CM collaborative. Support Care Cancer. 2018;26(2):375-391; doi: 10.1007/s00520-017-3908-0.

11. Le A, Mitchell H-R, Zheng DJ, Rotatori J, Fahey JT, Ness KK, et al. A home-based physical activity intervention using activity trackers in survivors of childhood cancer: a pilot study. Pediatr Blood Cancer. 2017;64(2): 387-394; doi: 10.1002/pbc.26235.

12. Lam KKW, Li WHC, Chung OK, Ho KY, Chiu SY, Lam HS, et al. An integrated experiential training programme with coaching to promote physical activity, and reduce fatigue among children with cancer: a randomised controlled trial. Patient Educ Couns. 2018;101(11):1947-1956; doi: 10.1016/j.pec.2018.07.008

13. Braam KI, van Dijk-Lokkart EM, Kaspers GJL, Takken T, Huisman J, Buffart LM, et al. Effects of a combined physical and psychosocial training for children with cancer: a randomized controlled trial. BMC Cancer. 2018;18(1): 1289; doi: 10.1186/s12885-018-5181-0.

14. Baker KS, Bresters D, Sande JE. The burden of cure: long-term side effects following hematopoietic stem cell transplantation (HSCT) in children. Pediatr Clin North Am. 2010;57(1):323-342; doi: 10.1016/j.pcl.2009.11.008.

15. Mohty B, Mohty M. Long-term complications and side effects after allogeneic hematopoietic stem cell transplantation: an update. Blood Cancer J. 2011;1(4):e16; doi: 10.1038/bcj.2011.14.

16. Ruble K, Krupski C, Chen A, Dandoy CE. Quality and safety in hematopoietic stem cell transplant patients. In: Dandoy CE, Hilden JM, Billett AL, Mueller BU (eds.), Patient safety and quality in pediatric hematology/oncology and stem cell transplantation. Cham: Springer; 2017; 297-324.

17. Felder-Puig R, di Gallo A, Waldenmair M, Norden $P$, Winter A, Gadner $\mathrm{H}$, et al. Health-related quality of life of pediatric patients receiving allogeneic stem cell or bone marrow transplantation: results of a longitudinal, multicenter study. Bone Marrow Transplant. 2006;38(2):119 126; doi: 10.1038/sj.bmt.1705417.

18. Ness KK, Hudson MM, Ginsberg JP, Nagarajan R, Kaste SC, Marina N, et al. Physical performance limitations in the Childhood Cancer Survivor Study cohort. J Clin Oncol. 2009;27(14):2382-2389; doi: 10.1200/JCO.2008.21.1482.

19. Tanzi EM. Health-related quality of life of hematopoietic stem cell transplant childhood survivors: state of the science. J Pediatr Oncol Nurs. 2011;28(4):191-202; doi: 10.1177/1043454211408100.

20. Van Haren IEPM, Timmerman H, Potting CM, Blijlevens NMA, Staal JB, Nijhuis-van der Sanden MWG. Physical exercise for patients undergoing hematopoietic stem cell transplantation: systematic review and meta-analyses of randomized controlled trials. Phys Ther. 2013;93(4): 514-528; doi: 10.2522/ptj.20120181.

21. Streckmann F, Kneis S, Leifert JA, Baumann FT, Kleber $\mathrm{M}$, Ihorst $\mathrm{G}$, et al. Exercise program improves therapy-related side-effects and quality of life in lymphoma patients undergoing therapy. Ann Oncol. 2014;25(2): 493-499; doi: 10.1093/annonc/mdt568.

22. Wiskemann J, Dreger P, Schwerdtfeger R, Bondong A, Huber G, Kleindienst N, et al. Effects of a partly self-administered exercise program before, during, and after allogeneic stem cell transplantation. Blood. 2011;117(9): 2604-2613; doi: 10.1182/blood-2010-09-306308.

23. Braam KI, van der Torre $P$, Takken T, Veening MA, van Dulmenden Broeder E, Kaspers GJL. Physical exercise training interventions for children and young adults during and after treatment for childhood cancer. Cochrane Data- base Syst Rev. 2013;4:CD008796; doi: 10.1002/146518 58.CD008796.pub2.

24. Knols RH, de Bruin ED, Shirato K, Uebelhart D, Aaronson NK. Physical activity interventions to improve daily walking activity in cancer survivors. BMC Cancer. 2010; 10:406; doi: 10.1186/1471-2407-10-406.

25. Rosenhagen A, Bernhörster M, Vogt L, Weiss B, Senn A, Arndt $S$, et al. Implementation of structured physical activity in the pediatric stem cell transplantation. Klin Padiatr. 2011;223(3):147-151; doi: 10.1055/s-0031-12 71782.

26. Weiß B. Quality of life and tumour-related fatigue in relation to sports activity in paediatric supportive therapy [in German]. Master's thesis. Darmstadt: TU Darmstadt; 2009.

27. Jung M, Höhne A, Varni J, Klingebiel T, Landenberger M. The measurement of fatigue in paediatric oncology. The first German version of the "PedsQL 3.0 Multidimensional Fatigue Scale" [in German]. Kind Jugendmed. 2009; 9(4):218-224; doi: 10.1055/s-0038-1629061.

28. Oberoi S, Robinson PD, Cataudella D, Culos-Reed SN, Davis $\mathrm{H}$, Duong $\mathrm{N}$, et al. Physical activity reduces fatigue in patients with cancer and hematopoietic stem cell transplant recipients: a systematic review and meta-analysis of randomized trials. Crit Rev Oncol Hematol. 2018;122: 52-59; doi: 10.1016/j.critrevonc.2017.12.011.

29. Witlox L, Hiensch AE, Velthuis MJ, Steins Bisschop CN, Los M, Erdkamp FLG, et al. Four-year effects of exercise on fatigue and physical activity in patients with cancer. BMC Med. 2018;16(1):86; doi: 10.1186/s12916-0181075-x.

30. Chamorro-Viña C, Valentín J, Fernández L, GonzálezVicent M, Pérez-Ruiz M, Lucía A, et al. Influence of a moderate-intensity exercise program on early NK cell immune recovery in pediatric patients after reduced-intensity hematopoietic stem cell transplantation. Integr Cancer Ther. 2017;16(4):464-472; doi:10.1177/1534735416679515. 